Original article

\title{
Problematic cellular phone use: a factor analysis
}

\author{
Saeideh Moslemizadeh, Ahmadipour Habibeh \\ Kerman University of Medical Sciences, Kerman, Iran
}

Received 03 January 2020, Revised 1 May 2020, Accepted 14 August 2020

C 2020, Russian Open Medical Journal

Abstract: Background - Increasing access to smartphones has led some researches to focus in this regard.

Objective - the current study aimed to determine psychometric properties of the Persian version of Problematic Cellular Phone Use Questionnaire (PCPU-Q) among medical student at Kerman University of Medical Sciences.

Material and Methods - A cross-sectional study carried out on 351 medical students. Data collected using a questionnaire including demographic data and the Persian versions of PCPU-Q. The higher score indicates a greater problem. Data analyzed by SPSS 20 and Lisrel 8.8. Cronbach's alpha and intraclass correlation coefficient (ICC) and exploratory/confirmatory factor analysis used for determining the reliability and construct validity of the questionnaire, respectively.

Results - One hundred and seventy-two $(49.0 \%)$ of the students had a problematic CPU. Cronbach's alpha and ICC were 0.8 and 0.91 , respectively. The model had acceptable goodness of fit indices in confirmatory factor analysis.

Conclusion - The Persian version of PCPU-Q had good psychometric properties and can be useful for screening of problematic CPU among Iranian youth.

Keywords: reliability, cell phone, validity, problem behavior.

Cite as Moslemizadeh S, Habibeh A. Problematic cellular phone use: a factor analysis. Russian Open Medical Journal 2021; 10: e0317.

Correspondence to Habiben Ahmadipour. Address: Social Determinants of Health Research Center, Institute for Futures Studies in Health, Kerman University of Medical Sciences, Kerman, Iran. Phone: 034-33257313, E-mail: address: ahmadipour@kmu.ac.ir.

\section{Introduction}

Nowadays, a mobile or cellular phone is an integral part of our lives and has led to a significant change in our daily communications. In the majority of countries, more than half of the population use cell phones and globally, an estimated 6.9 billion subscriptions reported in 2014 [1, 2]. Among the youth, especially university students, access to the cellular phone is felt like a necessity $[3,4]$.

In recent years, high accessibility of smartphones has led to further research on smartphone addiction and its consequences $[5,6]$. Several complications such as depression, anxiety, and low self-esteem are attributable to problematic cell phone use (CPU) $[7,8]$. Also, internet related disorders have been listed in the research criteria of the Diagnostic and Statistical Manual of Mental Disorders (DSM-VI) [5].

Several instruments have been developed to evaluate behavioral and functional disorders associated with using a cellular phone. Some of them such as Cellular Phone Dependency Questionnaire (CPDQ), Mobile Phone Problematic Use Scale (MPPUS), Problematic Mobile Phone Use Questionnaire (PMPUQ), Mobile Phone Dependency (MDS), and mobile phone addiction index (MPAl) have been assessed in terms of their psychometric properties in different population including Iranians [3, 4, 9-11].

The 12-item Problematic Cellular Phone Use Questionnaire (PCPU-Q) was developed by Cheng-Fang Yen and colleagues to assess the presence of problematic CPU symptoms [12].
Given that, according to our literature review, this questionnaire has not been evaluated in Iranian population and on the other hand, due to its acceptable validity and reliability, along with fewer number of questions compared to other instruments, the current study aimed to determine the psychometric properties of the Persian version of PCPU-Q among medical student at Kerman University of Medical Sciences (Kerman, Iran).

\section{Material and Methods}

\section{Subjects}

A cross-sectional study carried out on 351 medical students at KUMS (Kerman, a province in southeastern of Iran).

The students who were studying at different educational levels (basic sciences, pathophysiology, clerkship, and internship] at KUMS between January and March 2019 selected through the quota sampling method. After obtaining informed consent, the participants entered the study. The questionnaires with more than $10 \%$ unanswered questions were excluded.

\section{Instruments}

The data collected using a self-administered questionnaire including demographic data, such as age, gender, and educational level, residence and marital status, and household monthly income and the Persian version of PCPU-Q. The first seven questions of the instrument determine the occurrence of problematic CPU 
symptoms in the previous year. The rest of the questions assess the functional impairment resulting from the problematic CPU including the academic, relationship with the family and friends, and physical, psychological and financial problems. Response to each question is on Yes/No scale and having a positive response in $\geq$ four items of the first seven questions indicate problematic CPU. The internal consistency using Cronbach's alpha and test-retest reliability of the tools was 0.85 and 0.410 to 0.778 , respectively [12].

To provide the Persian version, after obtaining the permission, forward and backward translation used and then the questionnaire adapted culturally. The reliability of the Persian version was determined 0.8 using Cronbach's Alfa coefficient. Also, the test-retest reliability of the instrument was determined 0.91 by intraclass correlation coefficient (ICC). To do so, 20 medical students completed the questionnaire twice at two- week intervals. To determined construct validity, principal component analysis (PCA) using Kaiser Criterion and confirmatory factor analysis employed. The questionnaires were completed anonymously and voluntarily. It took about eight to ten minutes to complete the questionnaire.

\section{Statistics analysis}

Data analyzed using SPSS software version 20.0 (SPSS Inc., Chicago, IL, USA) and LISREL version 8.80 (Scientific Software International, Chicago, IL, USA). Chi-square and logistic regression applied.

\section{Results}

Of 351 medical students who participated in the current study, the majority of them (74.9\%) were female, single $(82.1 \%)$, in the basic sciences level (39.9\%), and living in the dormitory (39.3\%). The mean age of participants was $22.3 \pm 2.7 \mathrm{yrs}$.

One hundred and seventy-two $(49.0 \%)$ of the participants had $\geq$ four positive response on the first seven questions indicating the presence of problematic CPU. In logistic regression, an only educational level significantly predicted the presence of problematic CPU. The odds of the problematic CPU was 0.47 times lower in those who were in the internship compared to clerkship. ( $\mathrm{OR}=0.47, \mathrm{Cl} 195 \%=0.22-0.90, \quad \mathrm{P}=0.04)$. Academic performance
(20.5\%) was the most common affected function due to problematic CPU. After that were dysfunction in relationship with the family (15.4\%), physical and psychological health (15.4\%), financial affairs (8.3\%), and Relationships with friends (7.7\%), respectively.

In exploratory factor analysis, Kaiser-Meyer-Olkin Measure (KMO) was 0.79 and Bartlett's test of sphericity was statistically significant $(P=0.001, \chi 2=741.4, d f=66)$ indicating the adequacy of sampling and justifiability of factor analysis. Table 1 shows the exploratory factor loading of the Persian version of PCPU-Q.

In confirmatory factor analysis, the model had acceptable goodness of fit indices $(\chi 2 / d f=5.01, R M S E A=0.11$, SRMR $=0.07$, $\mathrm{GFI}=0.89, \mathrm{AGFI}=0.83, \mathrm{CFI}=0.84, \mathrm{IFI}=0.84$, and $\mathrm{NNFI}=0.81$ ).

\section{Discussion}

Our study revealed that the Persian version of 12 -item Problematic Cellular Phone Use Questionnaire (PCPU-Q) had good psychometric properties. The reliability of it in terms of internal consistency and ICC reached an excellent level and both PCA and confirmatory factor analysis indicated good construct validity for it. Therefore, the instrument can be used as a simple, valid and reliable tool for screening of the problematic CPU symptoms among Iranian youth including university students. Cheng-Fang Yen and colleagues reported acceptable validity and reliability for the original version [12]. Based on our literature review, no study found which has examined the questionnaire in another language or population.

We found that near to half of our medical students reported $\geq$ four symptoms of problematic cellular phone use which was much higher than ones $(16.7 \%)$ reported by Yen and Yang among adolescents in Taiwan $[8,12]$. Problematic Smart Mobile Phone Usage was reported in 50.6 percent of adolescents aged 12-18 years in Turkey that is more compatible with our study [13].

We found no statistically significant difference in problematic CPU symptoms according to the demographic data which was consistent with the study done by Rupani Mihir et al in India [14]. Yen et al found neither age nor residential area had a relationship with problematic CPU but female adolescences were more likely to do so [12].

Table 1. Exploratory factor loading of the Persian version of PCPU-Q

\begin{tabular}{|c|c|c|c|}
\hline Number & Items & Factor & Factor loading \\
\hline 1 & To achieve satisfaction, I need to significantly increase the time and frequency of using my cell phone. & 1 & 0.30 \\
\hline 2 & I'm anxious and worried without using a cell phone & 1 & 0.40 \\
\hline 3 & Always use my phone much longer or more often than I want & 1 & 0.52 \\
\hline 4 & I have a constant tendency to reduce the use of mobile phones, but my efforts are unsuccessful & 1 & 0.42 \\
\hline 5 & I spend a lot of time using my mobile phone & 1 & 0.60 \\
\hline 6 & For the sake of using mobile phones, I cut my leisure, education and social activities and even let them go. & 1 & 0.54 \\
\hline 7 & $\begin{array}{l}\text { Despite the fact that I know that excessive use of mobile phones can lead to physical and psychological } \\
\text { complications, I will continue to use my phone over and over again. }\end{array}$ & 1 & 0.55 \\
\hline 8 & Because of the use of mobile phones, I have a weak relationship with friends or classmates & 1 & 0.42 \\
\hline 9 & I have a weak relationship with my family because of the use of mobile phones & 1 & 0.46 \\
\hline 10 & I have poor academic performance due to the use of mobile phones & 1 & 0.52 \\
\hline 11 & My physical and mental health has been compromised due to the use of mobile phones & 1 & 0.52 \\
\hline 12 & Because the cost of using a mobile phone, I found financial problems & 1 & 0.32 \\
\hline
\end{tabular}


In terms of the educational level, problematic CPU symptoms were lower in the internship. Interns are likely to have less chance of using mobile phones because of more engagement with patient care and night shifts.

Our study revealed that academic dysfunction was the most common reported functional impairment due to problematic cellular phone use which is compatible with Taiwanese study [12]. Similar studies in this field revealed an association of the problematic cell phone use and different physical and psychosocial impairments. Dey et al found problematic smartphone use (PSU) is associated with social anxiety and high-risk behaviors such as risky drinking [7]. Another study reported aggression, sleep disorders, low self-esteem, and even suicidal tendencies as the consequences of problematic smartphone use [8].

On the other hand, Elhai and colleagues discussed a phenomenon in which depressed and anxious college students are prone to have problematic smartphone use to relieve their boredom [15].

Maybe it does not matter that problematic cell phone use results in different physical and psychosocial impairments or vice versa, what is so important is that the symptoms should be timely identified and intervened to prevent serious complications among the youth (most affected group). This is even more important for medical students as future physicians.

Given the young structure of our population in Iran, since 2014 , integrated management of the age group 18 to 29 years has been considered in the primary health care system [16]. Therefore, there is an opportunity for health care providers, in addition, to provide routine care, the symptoms of problematic CPU assessed by a valid instrument. The Persian version PCPU-Q due to acceptable psychometric properties and also the fewer number of the items compared to other tools can be helpful in this regard.

In the current research, a cross-sectional method with its potential limitation in time measurement was used. However, this method is suitable for determining the psychometric properties of an instrument. On the other hand, the Persian version of PCPU-Q has been assessed for the first time among medical students who can represent the young Iranian population.

\section{Conclusion}

The prevalence of problematic CPU symptoms was higher among our medical students and given that academic dysfunction was the most common reported consequences, it is necessary to consider timely screening in the periodic evaluation of students. Since that, the Persian version of PCPU-Q had good psychometric properties, it can be helpful for such assessments.

\section{Limitations}

A cross-sectional method was used with its potential limitation and the data was self-reported, which does not necessarily yield precise evidence.

\section{Acknowledgment}

The author express their gratitude to all medical students whom their cooperation, made this study possible.

\section{Conflicts of interest}

Both authors declare that there were no conflicts of interest.

\section{Ethical approval}

Our study approved by the Ethics Committee of Kerman University of Medical Sciences (Code: IR.KMU.REC.1396.2064). The study have been performed in accordance with the ethical standards as laid down in the 1964 Declaration of Helsinki and its later amendments.

\section{References}

1. Mohammadi M, Alavi SS, Farokhzad P, Jannatifard F, Mohammadi Kalhori S, Sepahbodi G, et al. The Validity and Reliability of the Persian Version Test of Mobile Phone Dependency (TMD). Iran J Psychiatry 2015; 10(4): 265-272. https://pubmed.ncbi.nlm.nih.gov/27006671.

2. World Health Organization. Electromagnetic fields and public health: mobile phones. 2014. https://www.who.int/en/news-room/factsheets/detail/electromagnetic-fields-and-public-health-mobilephones.

3. Mohammadi Kalhori S, Mohammadi MR, Alavi SS, Jannatifard F, Sepahbodi G, Baba Reisi M, et al. Validation and Psychometric Properties of Mobile Phone Problematic Use Scale (MPPUS) in University Students of Tehran. Iran J Psychiatry 2015; 10(1): 25-31. https://pubmed.ncbi.nlm.nih.gov/26005477.

4. Mazaheri MA, Karbasi M. Validity and reliability of the Persian version of mobile phone addiction scale. J Res Med Sci 2014; 19(2): 139-144. https://pubmed.ncbi.nlm.nih.gov/24778668.

5. Lin $\mathrm{YH}$, Chiang $\mathrm{CL}$, Lin $\mathrm{PH}$, Chang $\mathrm{LR}$, Ko $\mathrm{CH}$, Lee $\mathrm{YH}$, et al. Proposed Diagnostic Criteria for Smartphone Addiction. PLoS One 2016; 11(11) e0163010. https://doi.org/10.1371/journal.pone.0163010.

6. Yang Z, Asbury K, Griffiths MD. An exploration of problematic smartphone use among Chinese university students: Associations with academic anxiety, academic procrastination, self-regulation and subjective wellbeing. Int J Ment Health Addiction 2018; 17(3): 596-614. https://doi.org/10.1007/s11469-018-9961-1.

7. Dey M, Studer J, Schaub MP, Gmel G, Ebert DD, Lee JY, et al. Problematic smartphone use in young Swiss men: its association with problematic substance use and risk factors derived from the pathway model. J Behav Addict 2019; 8(2): 326-334. https://doi.org/10.1556/2006.8.2019.17.

8. Yang YS, Yen JY, Ko CH, Cheng CP, Yen CF. The association between problematic cellular phone use and risky behaviors and low selfesteem among Taiwanese adolescents. BMC Public Health 2010; 10 217. https://doi.org/10.1186/1471-2458-10-217.

9. Alavi SS, Maracy MR, Jannatifard F, Ojaghi R, Rezapour H. The psychometric properties of cellular phone dependency questionnaire in students of Isfahan: A pilot study. J Educ Health Promot 2014; 3: 71. https://doi.org/10.4103/2277-9531.134822.

10. Foerster M, Roser K, Schoeni A, Röösli M. Problematic mobile phone use in adolescents: derivation of a short scale MPPUS-10. Int J Public Health 2015; 60(2): 277-286. https://doi.org/10.1007/s00038-0150660-4.

11. Lopez-Fernandez O, Honrubia-Serrano L, Freixa-Blanxart M, Gibson W. Prevalence of problematic mobile phone use in British adolescents. Cyberpsychol Behav Soc Netw 2014; 17(2): 91-98. https://doi.org/10.1089/cyber.2012.0260.

12. Yen CF, Tang TC, Yen JY, Lin HC, Huang CF, Liu SC, et al. Symptoms of problematic cellular phone use, functional impairment and its association with depression among adolescents in Southern Taiwan. J Adolesc 2009; 32(4): 863-873. https://doi.org/10.1016/j.adolescence.2008.10.006.

13. Fırat S, Gül H, Sertçelik M, Gül A, Gürel Y, Kılıç BG. The relationship between problematic smartphone use and psychiatric symptoms among adolescents who applied to psychiatry clinics. Psychiatry Res 2018; 270: 97-103. https://doi.org/10.1016/j.psychres.2018.09.015.

14. Rupani M, Parikh K, Trivedi A, Singh M, Patel A, Vadodariya B, et al. Cross sectional study on mobile phone involvement among medical students of a tertiary care teaching hospital of western India. Natl $J$ 
Community Med 2016; 7(6): 609-613. http://njcmindia.org/uploads/77 609-613.pdf.

15. Elhai JD, Vasquez JK, Lustgarten SD, Levine JC, Hall BJ. Proneness to boredom mediates relationships between problematic smartphone use with depression and anxiety severity. Social Science Computer Review 2018; 36(6): 707-720. https://doi.org/10.1177\%2F0894439317741087.

16. Ministry of Health. Care for the age group 18 to 29 years; for physician. 2014.(Persian).

https://phc.umsu.ac.ir/index.aspx?fkevid=\&siteid=9\&pageid=7072.

Authors:

Saeideh Moslemizadeh - General Practitioner, community and family medicine department, school of medicine, Kerman University of Medical Sciences, Kerman, Iran. https://orcid.org/0000-0002-9264-8861.

Ahmadipour Habibeh - associate professor of community medicine, Social Determinants of Health Research Center, Institute for Futures Studies in Health, Kerman University of Medical Sciences, Kerman, Iran. http://orcid.org/0000-0001-5880-451X. 